\title{
RVG-modified exosomes derived from mesenchymal stem cells rescue memory deficits by regulating inflammatory responses in a mouse model of Alzheimer's disease
}

Guo-hong Cui ${ }^{1 \dagger}$, Hai-dong Guo ${ }^{2 \dagger}$, Han $\mathrm{Li}^{2+}$, Yu Zhai ${ }^{1}$, Zhang-bin Gong ${ }^{3}$, Jing Wu', Jian-sheng Liu', You-rong Dong ${ }^{1}$, Shuang-xing $\mathrm{Hou}^{4 *}$ and Jian-ren Liü ${ }^{*}$

\begin{abstract}
Background: Exosomes are lipid-bilayer enclosed nano-sized vesicles that transfer functional cellular proteins, mRNA and miRNAs. Mesenchymal stem cells (MSCs) derived exosomes have been demonstrated to prevent memory deficits in the animal model of Alzheimer's disease (AD). However, the intravenously injected exosomes could be abundantly tracked in other organs except for the targeted regions in the brain. Here, we proposed the use of central nervous system-specific rabies viral glycoprotein (RVG) peptide to target intravenously-infused exosomes derived from MSCs (MSC-Exo) to the brain of transgenic APP/PS1 mice. MSC-Exo were conjugated with RVG through a DOPE-NHS linker.

Results: RVG-tagged MSC-Exo exhibited improved targeting to the cortex and hippocampus after being administered intravenously. Compared with the group administered MSC-Exo, in the group administered RVGconjugated MSC-Exo (MSC-RVG-Exo) plaque deposition and A $\beta$ levels were sharply decreased and activation of astrocytes was obviously reduced. The brain targeted exosomes derived from MSCs was better than unmodified exosomes to improve cognitive function in APP/PS1 mice according to Morris water maze test. Additionally, although MSC-Exo injected intravenously reduced the expression of pro-inflammatory mediators TNF- $\alpha$, IL- $\beta$, and IL6 , but the changes of anti-inflammatory factors IL-10 and IL-13 were not obvious. However, administration of MSCRVG-Exo significantly reduced the levels of TNF- $a, I L-\beta$, and IL-6 while significantly raised the levels of IL-10, IL-4 and IL-13.

Conclusions: Taken together, our results demonstrated a novel method for increasing delivery of exosomes for treatment of AD. By targeting exosomes to the cortex and hippocampus of AD mouse, there was a significant improvement in learning and memory capabilities with reduced plaque deposition and A levels, and normalized levels of inflammatory cytokines.
\end{abstract}

Keywords: Alzheimer's disease, Exosomes, Targeting, Mesenchymal stem cells, Inflammatory cytokine

\footnotetext{
* Correspondence: housx021@163.com; liujr021@vip.163.com

† Guo-hong Cui, Hai-dong Guo and Han Li contributed equally to this work.

${ }^{4}$ Department of Neurology, Shanghai Pudong Hospital, Fudan University

Pudong Medical Center, Shanghai 201399, China

'Department of Neurology, Shanghai No. 9 People's Hospital, Shanghai

Jiaotong University School of Medicine, Shanghai 200011, China

Full list of author information is available at the end of the article
}

(c) The Author(s). 2019 Open Access This article is distributed under the terms of the Creative Commons Attribution 4.0 International License (http://creativecommons.org/licenses/by/4.0/), which permits unrestricted use, distribution, and

reproduction in any medium, provided you give appropriate credit to the original author(s) and the source, provide a link to the Creative Commons license, and indicate if changes were made. The Creative Commons Public Domain Dedication waiver (http://creativecommons.org/publicdomain/zero/1.0/) applies to the data made available in this article, unless otherwise stated. 


\section{Introduction}

Alzheimer's disease (AD), the most prevalent type of dementia, is characterized by excessive deposition of amyloid- $\beta$ peptide $(A \beta)$ and neurofibrillary tangles, which cause neuronal dysfunction and loss ultimately [1]. Rapid advances in stem-cell biology have provided a promising novel therapy for $\mathrm{AD}$. A huge volume of data has demonstrated that stem cell transplantation improved cognition, learning, and memory by replacement of damaged neurons, release of neurotrophic factors, and clearance of $A \beta$ plaques [2]. Our previous study showed that transplantation of neural stem cells (NSCs) improved cognitive function in an $\mathrm{A} \beta$-infused model of $A D$ via neuron differentiation and paracrine action [3]. In addition, chronic inflammation is involved in the pathogenesis of $\mathrm{AD}$, and mesenchymal stem cells (MSCs) appear to exert anti-inflammatory effects by modulating the balance of proinflammatory and anti-inflammatory factors [4].

Recent data showed that stem cell-derived exosomes might be a new strategy for several central nervous system (CNS) disorders. The exosomes are small biological lipid membrane vesicles with diameters ranging from $30 \sim 100 \mathrm{~nm}$ found in various cell types and the major mediators of intercellular communication through shuttling biologically active molecules to targeted recipient cells [5]. The transfer of exosomal cargo provides a rationale for the many reported stem cell-based therapeutic outcomes. For example, MSC-derived exosomes benefit neurite remodeling and functional recovery after stroke by mediating the miR-133b transfer to astrocytes and neurons [6]. Moreover, it has been reported that human adipose MSC-derived exosomes contain functional neprilysin, a major $A \beta$-degrading enzyme and, thus, have the potential to reduce the pathological accumulation of $\mathrm{A} \beta$ in $\mathrm{AD}$ [7]. In our previous study, the expression of miR-21 in MSC-derived exosomes was involved in modulation of the inflammatory response and improvement of cognitive function in an APP/PS1 double transgenic model of AD [8]. In addition, miRNA-181c expression in MSC-exosomes reduced burn-induced inflammation by down-regulating the toll-like receptor 4 signaling pathway [9].

Although the exosomes have the ability to cross the blood-brain barrier (BBB), a recent dynamic biodistribution study of exosomes revealed a significant amount of exosomes accumulated in the spleen and liver, and very limited exosomes signals were detected in the brain after systemic administration [10]. Thus, we propose that surface modified stem cell-derived exosomes targeted to the brain are agents with superior therapeutic potential to prevent memory deficits in AD. The CNS-specific rabies viral glycoprotein (RVG) peptide interacts specifically with the acetylcholine receptor to enable viral entry into neuronal cells [11]. Lydia et al. acquired targeted exosomes by engineering the exosomes to express RVG peptide and intravenous injection of these engineered exosomes delivered siRNA specifically to the mouse brain [12]. Furthermore, the targeted RVG exosomes loaded with opioid receptor $\mathrm{Mu}$ (MOR) siRNA was a potential novel strategy for the treatment of morphine relapse by down-regulating MOR expression levels [13].

In this study, we created brain-targeting exosomes derived from MSCs, through the use of peptide RVG, to increase the efficacy of intravenously delivered exosomes. Then the RVG modified exosomes were intravenously administrated to APP/PS1 transgenic mice through the tail vein to observe its therapeutic effect through Morris water maze test. Plaque deposition and A $\beta$ accumulation were detected through Thioflavin-S staining and ELISA, respectively. Additionally, the expression of pro-inflammatory and anti-inflammatory cytokines were determined through quantitative real-time polymerase chain reaction (qRT-PCR) and ELISA. This study presented a feasible way to develop exosomes targeted to the brain for treating AD.

\section{Materials and methods \\ Animals}

APP/PS1 double transgenic mice, B6C3-Tg (APPswe, PSEN1dE9) 85Dbo/J (original species No. 004462), were obtained from Jackson Laboratories (Bar Harbor, ME). The transgenic mice were maintained on a standard 12-h light/dark cycle at a constant temperature, with free access to food and water. This study was approved by the animal ethics committee of Shanghai University of TCM and the Animal Research Committee of Shanghai. All of the protocols were based on the "Guide for the Care and Use of Laboratory Animals" of the National Institutes of Health (USA) and efforts were made to minimize the number of animals used and any discomfort experienced.

\section{Isolation and culture of MSCs}

To isolate bone marrow, mice were killed by cervical dislocation and the hind limbs were dissected from the trunk of the body with care not to damage the femur. Bisect each hind limb by cutting through the knee joint. Remove the muscle and connective tissue from both the tibia and the femur. Cut the ends of the tibia and femur just below the end of the marrow cavity and flush the marrow using a 27-gauge needle attached to a 10-ml syringe containing Dulbecco's Modified Eagle's medium (DMEM, Gibco, San Diego, USA). The medium with bone marrow was centrifuged and the cell pellet was resuspended in DMEM supplemented with 15\% fetal bovine serum (FBS, Gibco) and then cultured at $37^{\circ} \mathrm{C}$ under a $5 \% \mathrm{CO}_{2}$ atmosphere. The medium was changed 
at $48 \mathrm{~h}$. For subculture, cells were detached with $0.25 \%$ trypsin-ethylene diamine tetra-acetic acid (Gibco Invitrogen, USA) and seeded in DMEM with 15\% FBS [14]. The characterization of cell surface markers (CD29, CD44, CD45) were detected through immunofluorescence staining. The cells were incubated with the primary antibodies (1:100, Abcam, USA) overnight at $4{ }^{\circ} \mathrm{C}$ after being fixed with $4 \%$ paraformaldehyde. Then the cells were labeled with Alexa Flour 488 secondary antibody (1:200, Invitrogen) for $2 \mathrm{~h}$ at room temperature.

\section{Isolation and injection of exosomes}

FBS was spun at $100,000 \mathrm{~g}$ for $2 \mathrm{~h}$ to avoid contamination by the FBS-derived exosomes before the experiment. The cell supernatant was collected and exosomes were isolated using gradient centrifugation as previously described [15]. In brief, the cell debris and large membrane vesicles were removed by sequential centrifugation at $300 \mathrm{~g}$ for $10 \mathrm{~min}, 2000 \mathrm{~g}$ for $10 \mathrm{~min}$, and $10,000 \mathrm{~g}$ for $30 \mathrm{~min}$, followed by filtration using $0.22 \mu \mathrm{m}$ syringe filters. Then, the cleared supernatant was transferred to a fresh tube and spun at 100,000 g for $70 \mathrm{~min}$. After that, the supernatant was removed completely and the pellet was washed with phosphate buffer solution (PBS) to collect the exosomes. The sizes and concentrations of exosomes were determined by nanoparticle tracking analysis (NTA; NanoSight, Malvern, Worcestershire, United Kingdom).

\section{Exosome tagging}

The exosomes were tagged with RVG peptide (YTIWMPENPRPGTPCDIFTNSRGKRASNG) shown to target the CNS. The peptide was custom synthesized and then was conjugated to the exosomes using modifications of previously described methods [16]. DOPE-NHS (dioleoylphosphatidylethanolamine N-hydroxysuccinimide; COATSOME $^{\bullet}$ FE-8181SU5, NOF America; White Plains, $\mathrm{NY)}$ and RVG peptide were combined and allowed to react for $1 \mathrm{~h}$ to obtain the DOPE-RVG. The DOPE-RVG was then incubated with the exosomes derived from MSCs. The DOPE-RVG to exosome labelling ratio was determined as 500:1 considering the length of peptide and radius of exosome. The modified exosomes subsequently were labelled with lipophilic dye DiI (1,1'-Dioctadecyl-3,3,3',3' -Tetramethylindocarbocyanine Perchlorate, ThermoFisher Scientific, Waltham, MA) for the biodistribution detection.

\section{Injection of exosomes}

The 7-month-old APP/PS1 mice were intravenously treated with PBS (AD), exosomes derived from MSCs (MSC-Exo) or RVG-conjugated MSC-Exo (MSC-RVG-Exo) monthly for 4 months (13 mice for each group). In all three groups, the injection volume was $100 \mu \mathrm{l}$. In both the MSC-Exo and
MSC-RVG-Exo treated groups, the treatments were $5 \times$ $10^{11}$ MSC-Exo or MSC-RVG-Exo in $100 \mu \mathrm{l}$ PBS, respectively. There was no drop-out during normal breading and the injection period. To detect the presence of the exosomes in the brain, 3 mice in each group were euthanized at $5 \mathrm{~h}$ after injection and the brain slides were examined by confocal microscopy after counterstained with 4',6-diamidino-2-phenylindole (DAPI).

\section{Behavior test}

The Morris water maze test was performed to ascertain the spatial memory performance after exosome administration. The water maze test includes spatial training and probe test. Briefly, the mice were placed into a black-painted circular water tank $(1.0 \mathrm{~m}$ diameter, $30 \mathrm{~cm}$ height) which contains a platform in the center of North-West quadrant (target quadrant). The platform was located about $1 \mathrm{~cm}$ beneath the water surface and different geometric shapes were positioned around the tank to provide spatial orientation. The mice were given $60 \mathrm{~s}$ to find this invisible platform in the first 5 days for spatial acquisition tests. Each mouse was placed in the water maze randomly from one of four different quadrants. The time to find the platform was recorded as the escape latency. Mice that did not find the platform within $60 \mathrm{~s}$ were manually guided to the platform by the experimenter. The animals underwent 4 trials per day and the swim traces were recorded by a video camera that was linked to a computer. In the post-training probe trial test, the platform was removed after training for 5 days. The mouse was placed in the opposite quadrant of the target quadrant in the maze, and allowed to swim for $60 \mathrm{~s}$ to assess learning and memory capabilities. The number of platform location crossings and time spent in the target quadrant were measured during this period.

\section{Tissue preparation and Thioflavin-S staining}

The mice were anesthetized via $1 \%$ pentobarbital sodium ( $45 \mathrm{mg} / \mathrm{kg}$, i.p.) after the behavior test and transcardially perfused with a $0.9 \%$ saline solution followed by $4 \%$ paraformaldehyde via the left ventricle. The brain tissue samples which cover the complete hippocampus were fixed with $4 \%$ paraformaldehyde at $4{ }^{\circ} \mathrm{C}$ overnight and embedded in Optimal Cutting Temperature (OCT; Sakura Finetek USA, Inc.) compound after osmotic dehydration in sucrose solutions. Sequential $12 \mu \mathrm{m}$ coronal sections were cut on a cryostat and stored at $-20{ }^{\circ} \mathrm{C}$ until staining. The sections were immersed in $0.5 \%$ Thioflavin-S (Sigma-Aldrich) dissolved in 50\% ethanol for $8 \mathrm{~min}$. Subsequently, the sections were washed three times with $50 \%$ ethanol and then twice in distilled water. The slides were mounted and images were acquired with a fluorescence microscope (IX53, Olympus). Five fields in the cortex and hippocampus of the mice were 
selected randomly and pixel areas of amyloid plaques were quantified using ImageJ software (NIH, Bethesda, MD, USA).

\section{Immunofluorescence staining}

After being permeabilized with $0.1 \%$ Triton X-100 and blocked with 5\% normal goat serum for $1 \mathrm{~h}$ at room temperature, the slides were incubated with rabbit anti-GFAP (1:200, Abcam, USA) overnight at $4{ }^{\circ} \mathrm{C}$. Corresponding secondary antibody as Alexa Fluor 488 (1:200, Invitrogen) was incubated for $2 \mathrm{~h}$ at room temperature. Five fields in each mouse were selected randomly and the fluorescence intensity was analyzed using Image-Pro Plus. The expression of GFAP was normalized to the values of $\mathrm{AD}$ group.

To examine the biodistribution of DiI-labeled exosomes in mouse brain, the brain slides were stained with DAPI, and images were taken using an immunofluorescence microscope. The mean fluorescence intensity was analyzed using Image-Pro Plus and the engraftment of DiI-labeled exosomes was normalized to the values of MSC-Exo group.

\section{Enzyme linked immunosorbent assay (ELISA)}

The concentration of soluble and insoluble $A \beta_{40}$ and $\mathrm{A} \beta_{42}$ in the brain was detected with ELISA analysis following the manufacturer's instructions of specific ELISA kits (Invitrogen, Camarillo, CA). The hippocampus and frontal cortex from one hemisphere were homogenized in ice-cold protein extraction buffer containing a protease inhibitor and centrifuged at $4{ }^{\circ} \mathrm{C}$ for $30 \mathrm{~min}$ to obtain the supernatants as the soluble extract. The protein concentration was quantified using a BCA protein assay (Thermo Fisher Scientific, Waltham, MA) for sample normalization. Detergent-insoluble $A \beta_{40}$ and $A \beta_{42}$ was detected by extracting pellets in $5 \mathrm{M}$ guanidine $\mathrm{HCl}$ buffer, followed by a 1:20 dilution in lysis buffer. Each experimental sample was run in duplicate, and the concentrations of $A \beta$ in sample were determined using the standard curve. Values are expressed as a relative \% of the AD group. The concentrations of pro-inflammatory or anti-inflammatory cytokines in the brain were also detected through ELISA (RayBiotech, GA, USA) according to the manufacturer's instructions.

\section{qRT-PCR}

Quantification of pro-inflammatory or anti-inflammatory gene expression was performed by qRT-PCR. The hippocampus and frontal cortex from one hemisphere were extracted and homogenized on ice immediately. Total RNA was extracted from the samples using TRIzol reagent (Invitrogen) according to the manufacturer's instructions. Subsequently, RNA samples $(0.8 \mu \mathrm{g})$ were reverse transcribed using QuantiTect Reverse
Transcription kit (Qiagen). Real-time PCR of cDNA was performed on ABI 7500 Real-Time PCR system (Applied Biosystems) using SYBR Green ready mix (Applied Biosystems). All reactions were done in triplicate. The relative expression levels were calculated according to the comparative CT method $\left(2^{-\Delta \Delta \mathrm{Ct}}\right)$.

\section{Statistical analysis}

All graphs are represented as the mean \pm standard deviation. The statistical significance was evaluated using Student's t-test when comparing with two groups. Multiple groups were compared using one-way ANOVA followed by least significant difference tests. Results were considered statistically significant when $P<0.05$. All statistical analysis was performed using SPSS 23.0 (SPSS Inc., USA).

\section{Results}

Engraftment of targeted exosomes derived from MSCs in the brain

The MSCs we harvested expressed CD29 and CD44 and were negative for CD45 (Additional file 1: Figure S1), which indicated that the purity of MSCs used in our study was high. To detect the presence of the exosomes derived from MSCs in the brain and whether RVG modification enhanced the engraftment of exosomes in the cortex and hippocampus, the slides of brain were observed under fluorescence microscope at $5 \mathrm{~h}$ after injection. The DiI-labeled exosomes were found in the cortex and hippocampus in both MSC-RVG-Exo group and MSC-Exo group. The number of injected exosomes was further compared by relative mean fluorescence intensity. Indeed, there was much more DiI-labeled exosomes in the cortex and hippocampus of the mice in the MSC-RVG-Exo group than that in the MSC-Exo group (Fig. 1a-d).

\section{Targeted exosomes derived from MSCs alleviated plaque deposition and $A \beta$ accumulation}

To investigate the effect of MSC-RVG-Exo on amyloid plaque loading in the brain, two experiments, including Thioflavin-S staining and ELISA were performed. MSC-Exo treatment decreased plaque deposition in the cortex according to Thioflavin-S staining and interestingly there was a dramatic reduction in plaque deposition in the cortex of mice treated with MSC-RVG-Exo compared with MSC-Exo injected mice (Fig. 2a, b). Similarly, plaque deposition in the hippocampus was significantly reduced after treated by MSC-Exo or MSC-RVG-Exo. Furthermore, the plaque deposition area in the hippocampus of MSC-RVG-Exo treated mice was much lower than that in mice injected with MSC-Exo (Fig. 2c, d). To further investigate whether MSC-RVG-Exo injection contributed to $A \beta$ clearance, the $A \beta$ content in the brain was examined 


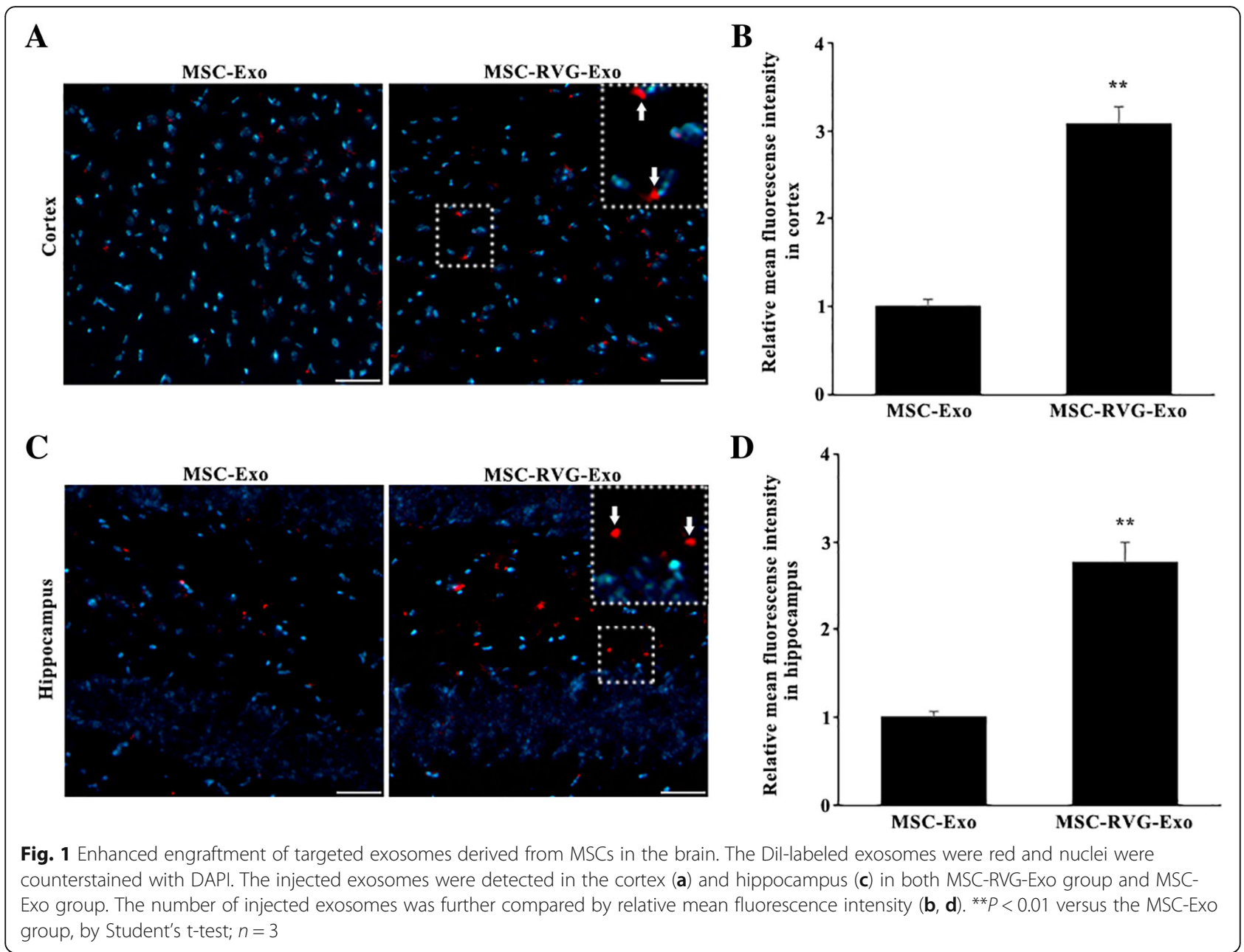

through an $A \beta$ ELISA kit. The data showed that treatment with MSC-Exo reduced both soluble $A \beta_{40}$ and soluble $A \beta_{42}$ in the brains of APP/PS1 mice. Compared with the group of mice treated by MSC-Exo, the concentration of soluble $\mathrm{A} \beta_{40}$ and soluble $A \beta_{42}$ was much lower in MSC-RVG-Exo treated mice (Fig. 2e, f). Additionally, the expression of insoluble $A \beta_{40}$ in the group of MSC-Exo was much lower than that in the group of saline-injected AD mice. No significant decrease in the expression of insoluble $A \beta_{42}$ was detected between the group treated with MSC-Exo and the group treated with saline. However, MSC-RVG-Exo treatment showed the lowest concentration of insoluble $A \beta_{40}$ and insoluble $A \beta_{42}$ in the brain and there was a significant difference between group MSC-RVG-Exo and MSC-Exo (Fig. 2g, h).

\section{Targeted exosomes derived from MSCs inhibited activation of astrocytes}

Next, the activation of astrocytes was analyzed according to immunofluorescence staining. As shown in Fig. 3a, the expression of GFAP, one marker of astrocytes, was highly robust in $\mathrm{AD}$ mice injected with saline. Injection of MSC-Exo and MSC-RVG-Exo could significantly attenuate the expression of GFAP in the brain. GFAP is just not an astrocyte marker, but also the marker of astrocyte activation because of the morphologic changes of astrocyte could be observed after immunostaining of GFAP [17-19]. The processes of astrocytes in AD group become thicker and the number of processes was increased when visualized with antibodies against GFAP. However, there were more astrocytes in the groups of exosome injection, especially the group of MSC-RVG-Exo, showed their original tiled domains. Statistically, the AD mice treated with MSC-RVG-Exo showed the lowest expression of GFAP among the groups (Fig. 3b).

\section{Targeted exosomes derived from MSCs ameliorated spatial learning and memory impairments in APP/PS1 mice}

Morris water maze test was performed to assess whether intravenous injection of brain targeted exosomes derived from MSCs could improve spatial learning and memory 
$\mathbf{A}$
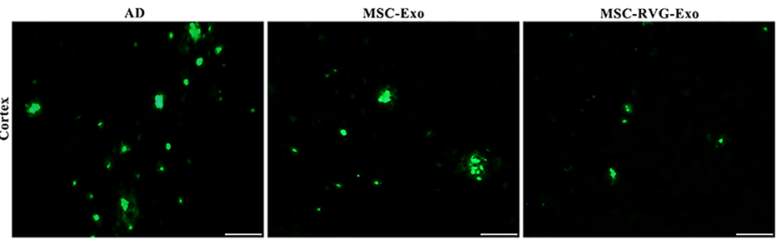

C

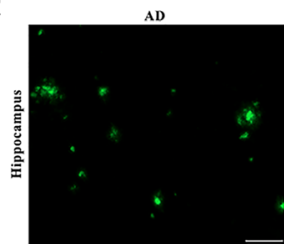

E

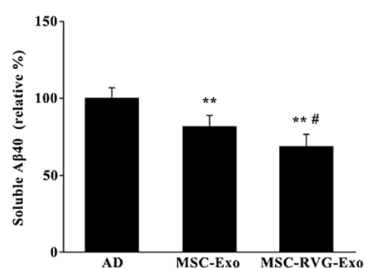

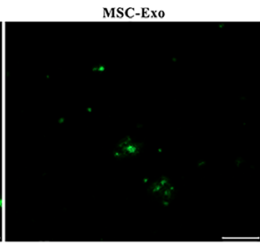

F

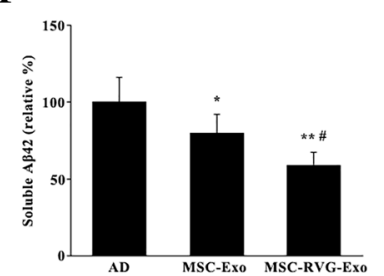

MSC-RVG-Exo

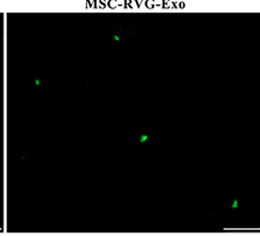

G

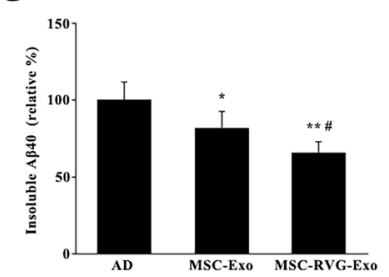

B

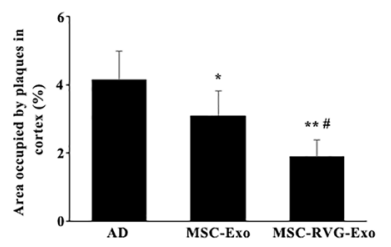

D

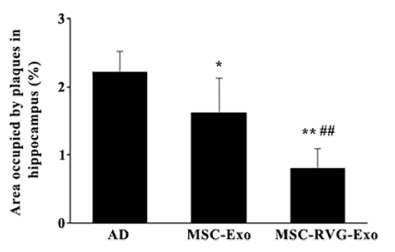

H

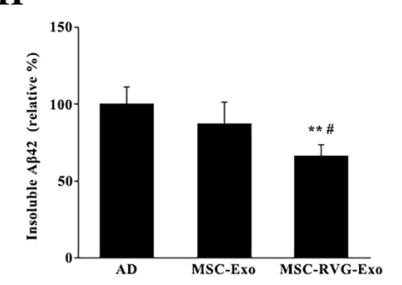

Fig. 2 Targeted exosomes derived from MSCS reduced plaques deposition and A 3 accumulation in the brain. Thioflavin-S staining was performed to detect the plaques in the cortex $(\mathbf{a}$, Scale bar $=50 \mu \mathrm{m})$ and quantitative analysis of the area occupied by plaques in the cortex (b) of APP/PS1 mice. The plaques deposition was also detected through Thioflavin-S staining in the hippocampus (c, Scale bar $=50 \mu m)$ and the area occupied by plaques in the hippocampus (d) was quantified. The plaque deposition area in the cortex and hippocampus of MSC-RVG-Exo treated mice were much lower than that in mice injected with MSC-Exo. The amount of soluble $A \beta 40(\mathbf{e})$ and $A \beta 42(\mathbf{f})$, and insoluble $A \beta 40(\mathbf{g})$ and $A \beta 42(\mathbf{h})$ in the brains were determined through a commercial ELISA kit. ${ }^{*} P<0.05$ and ${ }^{* *} P<0.01$ versus the AD group. $\# P<0.05$ and \#\#P<0.01 versus the MSC-Exo group, by one-way ANOVA; $n=5$

in APP/PS1 mice. As shown by mean escape latency during the acquisition training phase, spatial learning and memory was dramatically impaired in the saline-injected AD mice. MSC-Exo treatment significantly reduced the escape latency compared with saline-injected $\mathrm{AD}$ mice on days 4 and 5. From 3 days after acquisition training, mice treated with MSC-RVG-Exo presented obvious improved ability of learning and memory. Interestingly, mice injected with MSC-RVG-Exo showed much lower escape latency than that in the mice treated by MSC-Exo on days 4 and 5 (Fig. 4a). The number of platform location crosses was higher and the time spent in the target quadrant was longer for MSC-Exo than saline-injected AD mice. Compared with the mice treated by MSC-Exo, injection of MSC-RVG-Exo increased the number of platform location crossings and time spent in the target quadrant (Fig. 4b, c). The swimming speeds were similar among the groups, which suggested that the improved ability of learning and memory in mice treated by MSC-RVG-Exo resulted from cognitive processes but not non-cognitive behavioral components (Fig. 4d). Thus, the brain targeted exosomes derived from MSCs was better than unmodified exosomes to improve cognitive function in APP/PS1 mice.
Targeted exosomes derived from MSCs decreased proinflammatory factors and increased anti-inflammatory factors

The neuroinflammatory responses play a major role in the development of $\mathrm{AD}$ pathologies, including the formation of neurotoxic amyloid plaque and cognitive deficits. The mRNA expression of pro-inflammatory and anti-inflammatory cytokines was determined through qRT-PCR. We found that that the pro-inflammatory mediators TNF- $\alpha$, IL- $\beta$, and IL- 6 were significantly downregulated in AD mouse brains after MSC-Exo treatment or MSC-RVG-Exo treatment. Compared with the group of MSC-Exo, the levels of TNF- $\alpha$ and IL-1 $\beta$ were much lower in the group treated with MSC-RVG-Exo (Fig. 5a-c). The expression of anti-inflammatory cytokines IL-10, IL-4 and IL-13 in the brain was only slightly upregulated after treated by MSC-Exo. However, MSC-RVG-Exo treatment significantly raised the level of IL-10, IL-4 and IL-13. Furthermore, the expression of IL-10 and IL-4 was significantly increased in MSC-RVG-Exo injected AD mice, compared with MSC-Exo injected AD mice (Fig. 5d-f). Moreover, the concentrations of TNF- $\alpha$, IL- $\beta$, IL- 6 and IL-4 determined by ELISA was similar to the expression patterns of mRNA 


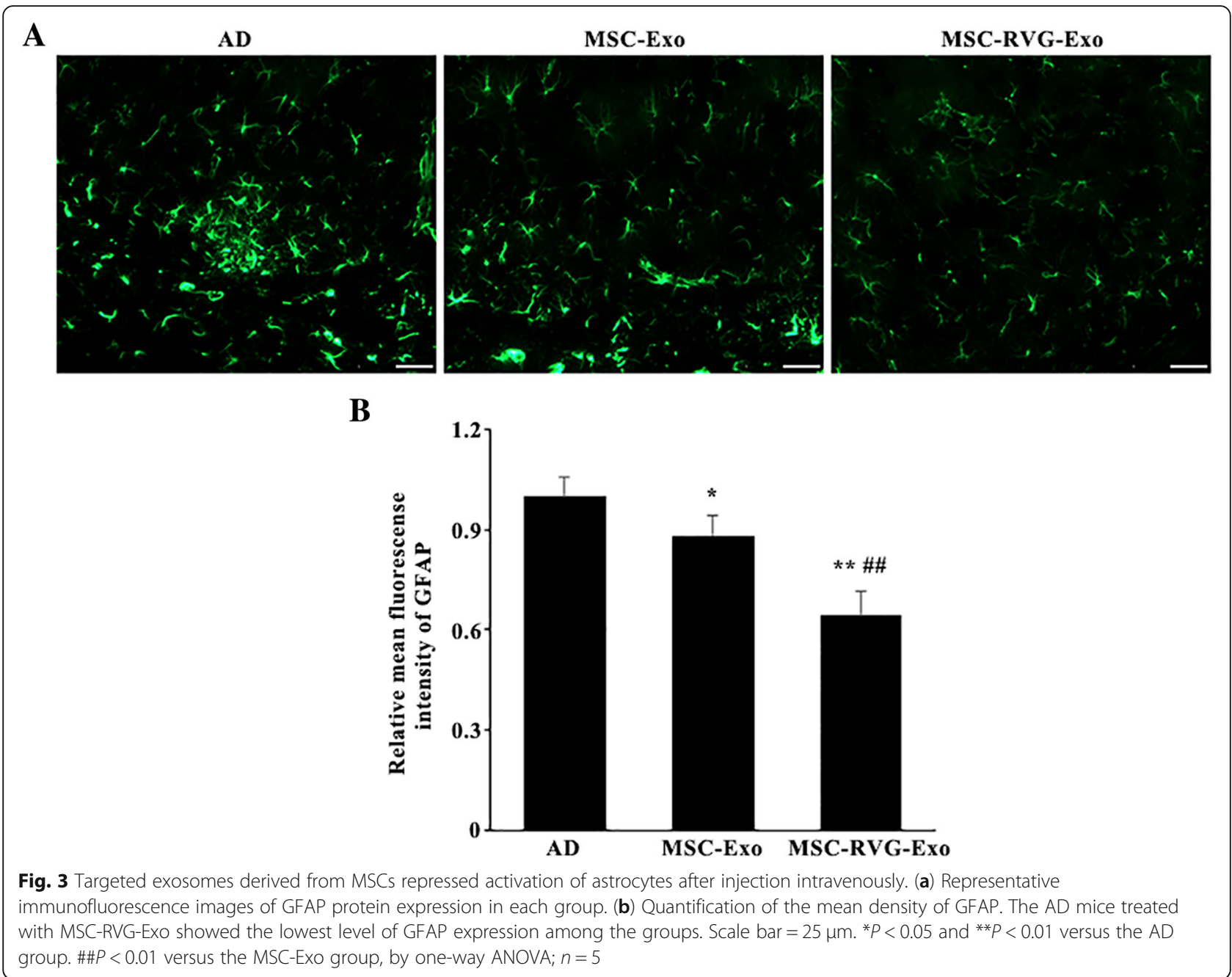

level (Fig. 5g-j). Thus it appears that MSC-RVG-Exo treatment could act as an important regulator of both the pro-inflammatory and anti-inflammatory responses in the AD brain.

\section{Discussion}

Currently, there are no treatments that halt or reverse the course of $\mathrm{AD}$, and only palliative therapies exist. In our previous study, we reported that the APP/PS1mice treated with exosomes from MSCs showed obviously improved learning and memory [8]. However, the intravenously injected exosomes could be obviously tracked in lungs and spleen. Thus, it is interesting to investigate the enhancement of learning and memory performance in $\mathrm{AD}$ mice by targeting exosomes to the brain. The present study firstly demonstrated an effective method for increasing the potency of MSC-Exo for the treatment of $\mathrm{AD}$ through modification with targeted peptide RVG. MSC-RVG-Exo administration significantly alleviated memory deficits and effectively decreased $A \beta$ levels and amyloid plaque loading in the brain of APP/PS1 mice.
Overall, successful development of brain targeted exosomes may lead to a viable therapy for patients with AD.

The viral entry into neuronal cells through the interaction between RVG and nicotinic acetylcholine receptor (AchR) on neuronal cells [20, 21]. It has been confirmed that the potential of RVG to mediate transvascular delivery of small interfering RNA (siRNA) to the CNS [22]. Recently, RVG has been engineered to exosomal surface via fused protein lysosome-associated membrane glycoprotein 2b (Lamp2b)-RVG in order to achieve neuron-specific targeting [12]. Furthermore, the modified exosomes, with RVG fused to exosomal protein Lamp2b, could efficiently deliver miR-124 to the infarct site after ischemia [23]. In our study, DiI fluorescence ferried by exosomes distributed in the brain, which was consistent with the work reported by Haney in 2015 [15]. However, compared with unmodified exosomes, the concentrated fluorescence in the cortex and hippocampus showed high targeting efficiency of MSC-RVG-Exo. It was reported that receptor-mediated transcytosis by means of the $\alpha 7$ subunit of the AchR was 


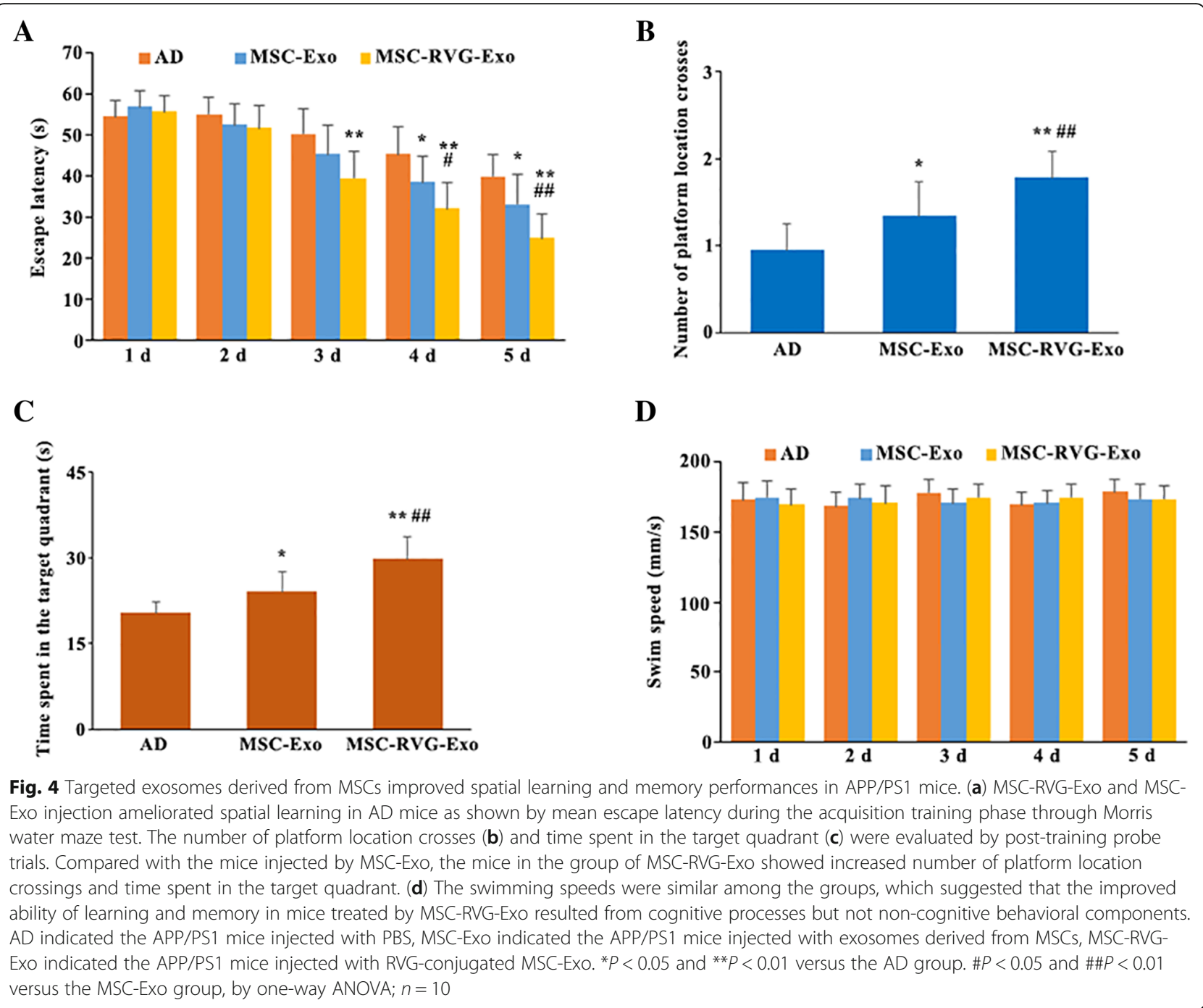

probably involved in the process of exosome invasion in the brain after intravenous injection. The $\alpha 7$ subtype, is highly expressed in brain regions relevant to cognitive and memory functions and involved in the pathophysiologic processes underlying AD [24]. However, the expressions of $\mathrm{AChRs}$ were significantly decreased in $\mathrm{AD}$ patients according to biochemical analyses of postmortem brains [25]. There was evidence that extended exposure times and high concentrations of $A \beta 42$ lead to dysregulation of $\alpha 7$ AChR, ERK MAPK, and CREB, as well as impaired memory and learning [26]. Besides, the significant decrease in $\alpha 7 \mathrm{AChR}$ protein expression takes place in the temporal cortex of AD brains. Thus, it's worthwhile to overcome this issue to optimize exosomal therapy for targeting the CNS in the future.

The use of DOPE-NHS allows for peptides to be conjugated to exosomes and possibly other membrane-based nanoparticles. In this study, MSC derived exosomes were conjugated with RVG through a DOPE-NHS linker. After being administered intravenously, RVG-tagged MSC-Exo exhibited improved targeting to the cortex and hippocampus. The mice treated by MSC-RVG-Exo showed alleviated spatial learning and memory impairments remarkably. Cardiac stem cell-derived exosomes were also conjugated with cardiac homing peptide (CHP) through this method to target intravenously-infused exosomes to the infarcted heart. The CHP exosomes reduced fibrosis and scar size, and increased cellular proliferation and angiogenesis after myocardial infarction [27]. Except the RVG system, other moieties including peptides, ligands or receptor-specific antibodies has also been utilized to achieve targeting delivery to the brain [28]. The cyclo (Arg-Gly-Asp-D-Tyr-Lys) peptide [c (RGDyK)] exhibits high affinity to integrin $\alpha_{\mathrm{v}} \beta_{3}$, where its expression on cerebral vascular endothelial cells is induced by ischemia $[29,30]$. Administration of c (RGDyK)-conjugated exosomes has resulted in a strong suppression of the inflammatory response and cellular apoptosis in the lesion 

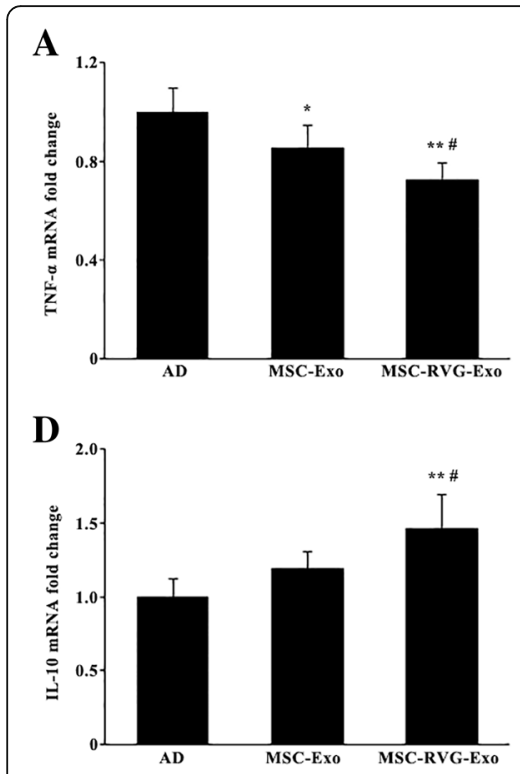

B

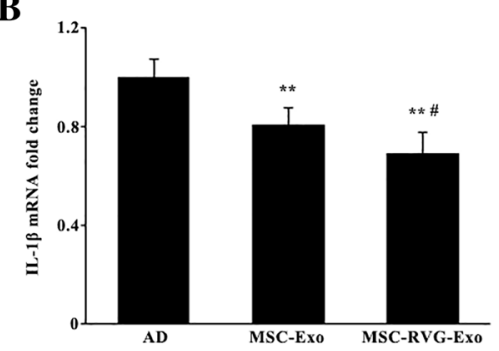

E

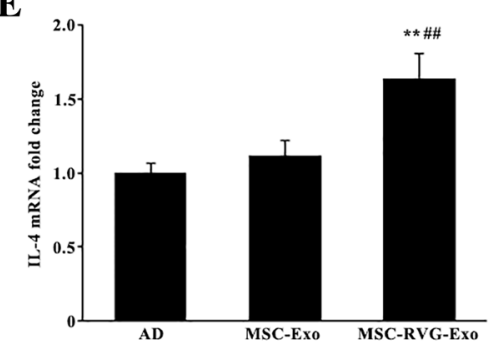

C

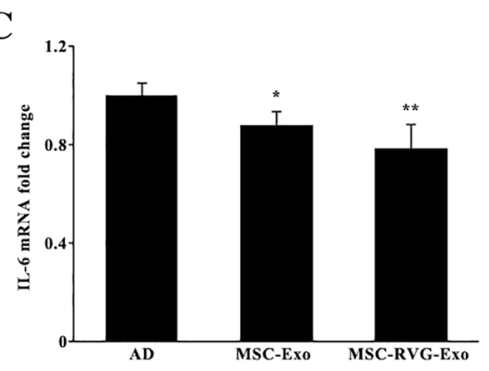

F

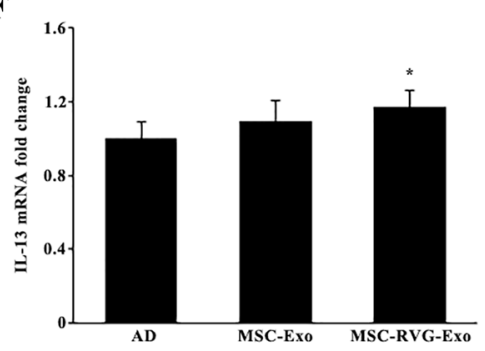

G

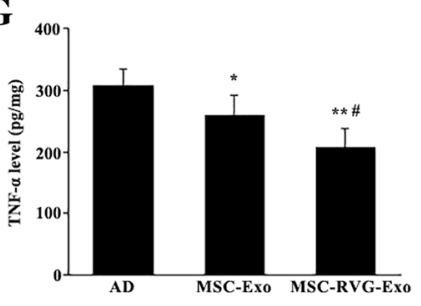

I

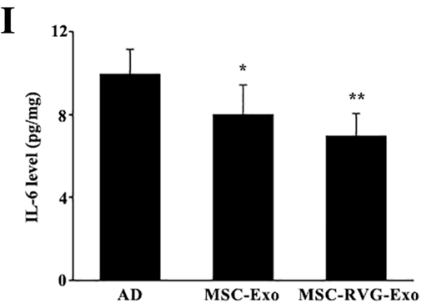

H

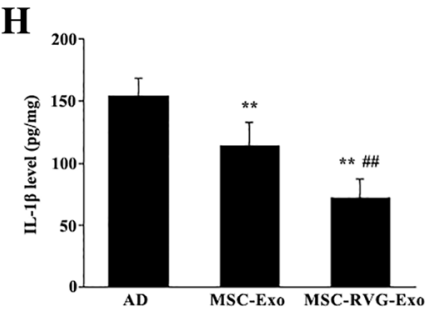

J

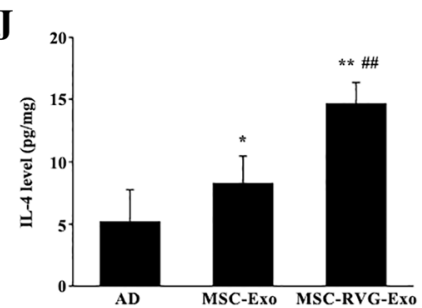

Fig. 5 Targeted exosomes derived from MSCs regulated the expression of pro-inflammatory and anti-inflammatory cytokines. The mRNA expression of pro-inflammatory and anti-inflammatory cytokines was detected by qRT-PCR. The pro-inflammatory mediators TNF-a, IL- $\beta$, and IL-6 were significantly downregulated in the groups of MSC-ExO and MSC-RVG-Exo. The levels of TNF- $a$ and IL-1 $\beta$ were much lower in the group of MSC-RVG-Exo than those in the group of MSC-Exo (a-c). After being treated by MSC-RVG-Exo, the mice showed elevated expression of anti-inflammatory cytokines IL-10, IL-4 and IL-13 (d-f). The concentrations of TNF- $\mathrm{a}, \mathrm{IL}-\beta, \mathrm{IL}-6$ and IL-4 in the brain were further detected through ELISA and the data showed that the concentrations of these cytokines was similar to the expression patterns of mRNA level ( $\mathbf{g}-\mathbf{j}) .{ }^{*} P<0.05$ and ${ }^{* *} P<0.01$ versus the $A D$ group. $\# P<0.05$ and $\# \# P<0.01$ versus the MSC-Exo group, by one-way ANOVA; $n=5$

region after cerebral ischemia [31]. The GE11 peptide has high affinity for the epidermal growth factor receptor and GE11-positive exosomes that contained the miRNA let-7 were able to prevent tumor growth [32]. In addition, there are other proteins which would be able to dock exosomes to certain cells, such as connexin 43 [33], connexin 26 [34] and tenascin C [35].

Exosomes are naturally occurring nanosized vesicles secreted by a variety of cells [36]. The exosomes play important roles in long-distance intercellular communications facilitating transfer of microRNAs, functional mRNAs and proteins [37]. The exosomes can avoid phagocytosis by macrophages due to the small size and be naturally stable to escape endosome/lysosome dependent degradation compared with liposomes and polymeric nanoparticles. Most importantly, accumulating evidences showed that exosomes could cross BBB which has been proven to be the major obstacle for delivery of drugs to the CNS. Recent findings 
revealed that communication mediated via exosomes is a major mediator of physiological and pathological processes in the CNS [38]. Compared with other kinds of cells, cultured MSC could produce exosomes in a larger scale [39]. It has been suggested that MSC-derived exosomes possess enzymatically active NEP and decreased both extracellular and intracellular A $\beta$ levels in N2a cells [7]. Indeed, intravenously injection of MSC derived exosomes sharply improved learning and memory of $\mathrm{AD}$ mice according to our previous work [8] and this study. However, as NSCs have been proven to have great potential to treat $\mathrm{AD}$ [3], it would be very interesting to compare the effects of exosomes from MSCs or NSCs on the improvement of learning and memory. At present, questions regarding the isolation and production processes, quality and quantity of exosomes impact the potency of exosome-based strategy remain unanswered. Besides, the dosage of exosome administered was from $10^{9}$ to $10^{12}$ according to the literatures and $5 \times 10^{11}$ exosomes were adopted in this study. The quantity and dosage of exosomes for the treatment of AD through intravenous injection should be further optimized. Despite these challenges, the emerging field of exosome-based therapy offers many opportunities for the treatment of $\mathrm{AD}$.

Inflammatory response has long been implicated in the process of neurodegeneration seen in $\mathrm{AD}$ sufferers [40]. TNF- $\alpha$, IL-1 $\beta$ and IL-6, as pro-inflammatory cytokines, showed a significant increase in AD mice and the levels of these cytokines may be directly related to the amount of soluble and insoluble $A \beta$ present in the brain [41]. The pleiotropic cytokines IL-4, IL-10 and IL-13, as anti-inflammatory factors, inhibit the synthesis and release of pro-inflammatory cytokines. IL-10 was found to suppress $A \beta$ and lipopolysaccharide-induced inflammatory proteins including TNF- $\alpha$, IL- $1 \beta$ and IL-6, while IL-4 and IL-13 reduced IL-6 secretion [42]. IL-4 also could attenuate the neuroinflammation induced by amyloid-beta in vivo and in vitro [43]. The nanomaterial, graphene quantum dots (GQDs) conjugated neuroprotective peptide glycine-proline-glutamate improved learning and memory capability, downregulated pro-inflammatory cytokines (TNF- $\alpha$, IL-1 $\beta$ and IL-6), and upregulated the anti-inflammatory cytokines (IL-4 and IL-10) in APP/PS1 transgenic mice [44]. The data presented in this study found the changes of anti-inflammatory factors were not obvious in the mice intravenously injected with unmodified exosomes from MSC. However, administration of MSC-RVG-Exo not only decreased the level of TNF- $\alpha$, IL- $1 \beta$ and IL- 6 , but also increased the level of IL-4, IL-10 and IL-13 in APP/PS1 mice. APP/PS1 mice began to exhibit elevated expression of GFAP from at least 7 months [45]. We also identified that injection of MSC-Exo and MSC-RVG-Exo could significantly attenuate the expression of GFAP. Moreover, the AD mice treated with MSC-RVG-Exo showed the lowest expression of GFAP. Upregulation of GFAP has been reported in animal models of $\mathrm{AD}$, which suggests that astrocytes are believed to play a central role in the pathogenesis of $\mathrm{AD}$ [46]. Astrocytes are involved in the development of $\mathrm{AD}$ through upregulating the expression of pro-inflammatory cytokines and chemokines as well as regulating the generation and degradation of $A \beta$ [47]. However, further investigations will reveal molecular mechanisms underlying the anti-inflammatory properties of exosomes derived from MSCs.

\section{Conclusions}

In summary, our current study demonstrated that the CNS-specific peptide RVG modified MSC-exosomes improved learning and memory function in APP/PS1 transgenic mice. In addition, our results showed that RVG modified MSC-exosomes could effectively reduce A $\beta$ accumulation and activation of glial cells, and balanced the inflammatory factors in the brain of AD mice. Thus, brain targeted exosomes derived from MSCs may rescue memory deficits by regulating inflammatory responses and well serve as a next generation drug for the treatment of $\mathrm{AD}$ and potentially for other neurodegenerative diseases.

\section{Additional file}

Additional file 1: Figure S1. The characteristics of MSCs was detemined by immunocytofluorescense. Most of the cells were postive for CD29 and CD44. Scale bar $=25 \mu \mathrm{m}$. (TIF $1360 \mathrm{~kb})$

\section{Abbreviations}

AD: Alzheimer's disease; $A \beta$ : amyloid- $\beta$ peptide; BBB: blood-brain barrier; CHP: cardiac homing peptide; CNS: central nervous system;

DMEM: Dulbecco's modified eagle's medium; FBS: fetal bovine serum;

MOR: opioid receptor Mu; MSC-Exo: exosomes derived from MSCs; MSC-RVGExo: RVG-conjugated MSC-Exo; MSCs: mesenchymal stem cells; NSCs: neural stem cells; PBS: phosphate buffer solution; RVG: rabies viral glycoprotein

\section{Acknowledgements}

Not applicable.

\section{Funding}

This work was supported by grants from the National Natural Science Foundation of China (31400838, 81673729 and 81774019), Program of No. 9 Shanghai People's Hospital (2013A05), Natural Science Foundation of Shanghai (No. 16ZR1437300), Outstanding Leaders Training Program of Pudong Health Bureau of Shanghai (PWR12015-06) and Shanghai Municipal Commission of Health and Family Planning, NO. ZY (2018-2020)-CCCX-2001-01.

\section{Availability of data and materials}

All data generated or analysed during this study are included in this published article.

\section{Authors' contributions}

Guo-hong Cui, Hai-dong Guo, Han Li, Zhang-bin Gong, Jing Wu, Jian-sheng Liu performed the experiments, analyzed and interpreted the data; Guohong Cui, Yu Zhai, You-rong Dong were major contributors in writing the manuscript; Shuang-xing Hou, Jian-ren Liu revised the manuscript. All authors read and approved the final manuscript. 


\section{Ethics approval and consent to participate}

This study was approved by the animal ethics committee of Shanghai University of TCM and the Animal Research Committee of Shanghai. All of the protocols were based on the "Guide for the Care and Use of Laboratory Animals" of the National Institutes of Health (USA) and efforts were made to minimize the number of animals used and any discomfort experienced.

\section{Consent for publication}

All participants have given consent for publication.

\section{Competing interests}

The authors have no conflicts of interest to disclose.

\section{Publisher's Note}

Springer Nature remains neutral with regard to jurisdictional claims in published maps and institutional affiliations.

\section{Author details}

'Department of Neurology, Shanghai No. 9 People's Hospital, Shanghai Jiaotong University School of Medicine, Shanghai 200011, China. ${ }^{2}$ Department of Anatomy, School of Basic Medicine, Shanghai University of Traditional Chinese Medicine, Shanghai 201203, China. ${ }^{3}$ Department of Biochemistry, School of Basic Medicine, Shanghai University of Traditional Chinese Medicine, Shanghai 201203, China. ${ }^{4}$ Department of Neurology, Shanghai Pudong Hospital, Fudan University Pudong Medical Center, Shanghai 201399, China.

\section{Received: 6 December 2018 Accepted: 26 April 2019} Published online: 13 May 2019

\section{References}

1. Wenk GL. Neuropathologic changes in Alzheimer's disease. J Clin Psychiatry. 2003;64:7-10.

2. Duncan T, Valenzuela M. Alzheimer's disease, dementia, and stem cell therapy. Stem Cell Res Ther. 2017;8:111.

3. Cui GH, Shao SJ, Yang JJ, Liu JR, Guo HD. Designer self-assemble peptides maximize the therapeutic benefits of neural stem cell transplantation for Alzheimer's disease via enhancing neuron differentiation and paracrine action. Mol Neurobiol. 2016;53:1108-23.

4. Lee HJ, Lee JK, Lee H, Carter JE, Chang JW, Oh W, Yang YS, Suh JG, Lee BH, Jin HK, et al. Human umbilical cord blood-derived mesenchymal stem cells improve neuropathology and cognitive impairment in an Alzheimer's disease mouse model through modulation of neuroinflammation. Neurobiol Aging. 2012;33:588-602.

5. Montecalvo A, Larregina AT, Shufesky WJ, Stolz DB, Sullivan ML, Karlsson JM, Baty CJ, Gibson GA, Erdos G, Wang Z, et al. Mechanism of transfer of functional microRNAs between mouse dendritic cells via exosomes. Blood. 2012;119:756-66.

6. Xin H, Li Y, Liu Z, Wang X, Shang X, Cui Y, Zhang ZG, Chopp M. MiR-133b promotes neural plasticity and functional recovery after treatment of stroke with multipotent mesenchymal stromal cells in rats via transfer of exosomeenriched extracellular particles. Stem Cells. 2013;31:2737-46.

7. Katsuda T, Tsuchiya R, Kosaka N, Yoshioka Y, Takagaki K, Oki K, Takeshita F, Sakai Y, Kuroda M, Ochiya T. Human adipose tissue-derived mesenchymal stem cells secrete functional neprilysin-bound exosomes. Sci Rep. 2013;3:1197.

8. Cui GH, Wu J, Mou FF, Xie WH, Wang FB, Wang QL, Fang J, Xu YW, Dong $Y R$, Liu JR, et al. Exosomes derived from hypoxia-preconditioned mesenchymal stromal cells ameliorate cognitive decline by rescuing synaptic dysfunction and regulating inflammatory responses in APP/PS1 mice. FASEB J. 2018;32:654-68.

9. Li X, Liu L, Yang J, Yu Y, Chai J, Wang L, Ma L, Yin H. Exosome derived from human umbilical cord mesenchymal stem cell mediates MiR-181C attenuating burn-induced excessive inflammation. EBioMedicine. 2016;8:72-82.

10. Lai CP, Mardini O, Ericsson M, Prabhakar S, Maguire C, Chen JW, Tannous BA, Breakefield XO. Dynamic biodistribution of extracellular vesicles in vivo using a multimodal imaging reporter. ACS Nano. 2014;8:483-94.

11. Lentz TL, Burrage TG, Smith AL, Crick J, Tignor GH. Is the acetylcholine receptor a rabies virus receptor? Science. 1982;215:182-4.
12. Alvarez-Erviti $L$, Seow $Y$, Yin $H$, Betts $C$, Lakhal $S$, Wood MJ. Delivery of siRNA to the mouse brain by systemic injection of targeted exosomes. Nat Biotechnol. 2011;29:341-5.

13. Liu Y, Li D, Liu Z, Zhou Y, Chu D, Li X, Jiang X, Hou D, Chen X, Chen Y, et al. Targeted exosome-mediated delivery of opioid receptor mu siRNA for the treatment of morphine relapse. Sci Rep. 2015;5:17543.

14. Hu Y, Lou B, Wu X, Wu R, Wang H, Gao L, Pi J, Xu Y. Comparative study on in vitro culture of mouse bone marrow mesenchymal stem cells. Stem Cells Int. 2018;2018:6704583.

15. Haney MJ, Klyachko NL, Zhao Y, Gupta R, Plotnikova EG, He Z, Patel T, Piroyan A, Sokolsky M, Kabanov AV, et al. Exosomes as drug delivery vehicles for Parkinson's disease therapy. J Control Release. 2015;207:18-30.

16. Kajimoto T, Okada T, Miya S, Zhang L, Nakamura S. Ongoing activation of sphingosine 1-phosphate receptors mediates maturation of exosomal multivesicular endosomes. Nat Commun. 2013;4:2712.

17. Pekny $M$, Wilhelmsson $U$, Pekna $M$. The dual role of astrocyte activation and reactive gliosis. Neurosci Lett. 2014;565:30-8.

18. Zanuzzi CN, Nishida F, Sisti MS, Barbeito CG, Portiansky EL. Reactivity of microglia and astrocytes after an excitotoxic injury induced by kainic acid in the rat spinal cord. Tissue Cell. 2019;56:31-40.

19. Wixey JA, Lee KM, Miller SM, Goasdoue K, Colditz PB, Tracey Bjorkman S, Chand KK. Neuropathology in intrauterine growth restricted newborn piglets is associated with glial activation and proinflammatory status in the brain. J Neuroinflammation. 2019;16(1):5.

20. Lafon M. Rabies virus receptors. J Neuro-Oncol. 2005;11:82-7.

21. Gastka M, Horvath J, Lentz TL. Rabies virus binding to the nicotinic acetylcholine receptor alpha subunit demonstrated by virus overlay protein binding assay. J Gen Virol. 1996;77(Pt 10):2437-40.

22. Kumar P, Wu H, McBride JL, Jung KE, Kim MH, Davidson BL, Lee SK, Shankar $P$, Manjunath $N$. Transvascular delivery of small interfering RNA to the central nervous system. Nature. 2007:448:39-43.

23. Yang J, Zhang X, Chen X, Wang L, Yang G. Exosome mediated delivery of miR-124 promotes neurogenesis after ischemia. Mol Ther Nucleic Acids. 2017;7:278-87.

24. Vallés AS, Borroni MV, Barrantes FJ. Targeting brain a7 nicotinic acetylcholine receptors in Alzheimer's disease: rationale and current status. CNS Drugs. 2014;28:975-87.

25. Perry EK, Perry RH, Smith CJ, Dick DJ, Candy JM, Edwardson JA, Fairbairn A, Blessed G. Nicotinic receptor abnormalities in Alzheimer's and Parkinson's diseases. J Neurol Neurosurg Psychiatry. 1987;50:806-9.

26. Dineley KT. Beta-amyloid peptide--nicotinic acetylcholine receptor interaction: the two faces of health and disease. Front Biosci. 2007;12:5030-8.

27. Vandergriff A, Huang K, Shen D, Hu S, Hensley MT, Caranasos TG, Qian L, Cheng K. Targeting regenerative exosomes to myocardial infarction using cardiac homing peptide. Theranostics. 2018;8:1869-78.

28. Huwyler J, Wu D, Pardridge WM. Brain drug delivery of small molecules using immunoliposomes. Proc Natl Acad Sci U S A. 1996;93:14164-9.

29. Guell K, Bix GJ. Brain endothelial cell specific integrins and ischemic stroke. Expert Rev Neurother. 2014;14:1287-92.

30. Haubner R, Wester HJ, Burkhart F, Senekowitsch-Schmidtke R, Weber W, Goodman SL, Kessler H, Schwaiger M. Glycosylated RGD-containing peptides: tracer for tumor targeting and angiogenesis imaging with improved biokinetics. J Nucl Med. 2001;42:326-36.

31. Tian T, Zhang HX, He CP, Fan S, Zhu YL, Qi C, Huang NP, Xiao ZD, Lu ZH, Tannous BA, et al. Surface functionalized exosomes as targeted drug delivery vehicles for cerebral ischemia therapy. Biomaterials. 2018; 150:137-49.

32. Ohno S, Takanashi M, Sudo K, Ueda S, Ishikawa A, Matsuyama N, Fujita K, Mizutani T, Ohgi T, Ochiya T, et al. Systemically injected exosomes targeted to EGFR deliver antitumor microRNA to breast cancer cells. Mol Ther. 2013; 21:185-91.

33. Chever O, Pannasch U, Ezan P, Rouach N. Astroglial connexin 43 sustains glutamatergic synaptic efficacy. Philos Trans R Soc Lond Ser B Biol Sci. 2014; 369:20130596.

34. Mercier F, Hatton Gl. Connexin 26 and basic fibroblast growth factor are expressed primarily in the subpial and subependymal layers in adult brain parenchyma: roles in stem cell proliferation and morphological plasticity? J Comp Neurol. 2001;431:88-104.

35. Kim MY, Kim OR, Choi YS, Lee H, Park K, Lee CT, Kang KW, Jeong S. Selection and characterization of tenascin C targeting peptide. Mol Cells. 2012;33:71-7. 
36. Vlassov AV, Magdaleno S, Setterquist R, Conrad R. Exosomes: current knowledge of their composition, biological functions, and diagnostic and therapeutic potentials. Biochim Biophys Acta. 2012;1820:940-8.

37. Valadi H, Ekstrom K, Bossios A, Sjostrand M, Lee JJ, Lotvall JO. Exosomemediated transfer of mRNAs and microRNAs is a novel mechanism of genetic exchange between cells. Nat Cell Biol. 2007;9:654-9.

38. Fruhbeis C, Frohlich D, Kramer-Albers EM. Emerging roles of exosomes in neuron-glia communication. Front Physiol. 2012;3:119.

39. Yeo RW, Lai RC, Zhang B, Tan SS, Yin Y, Teh BJ, Lim SK. Mesenchymal stem cell: an efficient mass producer of exosomes for drug delivery. Adv Drug Deliv Rev. 2013;65:336-41.

40. Cai Z, Hussain MD, Yan L. Microglia, neuroinflammation, and beta-amyloid protein in Alzheimer's disease. Int J Neurosci. 2014;124:307-21.

41. Patel NS, Paris D, Mathura V, Quadros AN, Crawford FC, Mullan MJ. Inflammatory cytokine levels correlate with amyloid load in transgenic mouse models of Alzheimer's disease. J Neuroinflammation. 2005;2:9.

42. Szczepanik AM, Funes S, Petko W, Ringheim GE. IL-4, IL-10 and IL-13 modulate a beta (1--42)-induced cytokine and chemokine production in primary murine microglia and a human monocyte cell line. J Neuroimmunol. 2001;113:49-62.

43. Lyons A, Griffin RJ, Costelloe CE, Clarke RM, Lynch MA. IL-4 attenuates the neuroinflammation induced by amyloid-beta in vivo and in vitro. J Neurochem. 2007;101:771-81

44. Xiao S, Zhou D, Luan P, Gu B, Feng L, Fan S, Liao W, Fang W, Yang L, Tao E, et al. Graphene quantum dots conjugated neuroprotective peptide improve learning and memory capability. Biomaterials. 2016:106:98-110.

45. Wang F, Zhong H, Li X, Peng Y, Kinden R, Liang W, Li X, Shi M, Liu L, Wang $Q$, et al. Electroacupuncture attenuates reference memory impairment associated with astrocytic NDRG2 suppression in APP/PS1 transgenic mice. Mol Neurobiol. 2014;50:305-13.

46. Rodríguez JJ, Olabarria M, Chvatal A, Verkhratsky A. Astroglia in dementia and Alzheimer's disease. Cell Death Differ. 2009;16:378-85.

47. Liu C, Cui G, Zhu M, Kang X, Guo H. Neuroinflammation in Alzheimer's disease: chemokines produced by astrocytes and chemokine receptors. Int J Clin Exp Pathol. 2014;7:8342-55.

Ready to submit your research? Choose BMC and benefit from:

- fast, convenient online submission

- thorough peer review by experienced researchers in your field

- rapid publication on acceptance

- support for research data, including large and complex data types

- gold Open Access which fosters wider collaboration and increased citations

- maximum visibility for your research: over $100 \mathrm{M}$ website views per year

At $\mathrm{BMC}$, research is always in progress.

Learn more biomedcentral.com/submissions 